\title{
A case of jugular paraganglioma presented as temporomandibular joint disorder
}

\begin{abstract}
Background: Jugular paraganlioma is a slow-growing tumor originating from the paraganglion cells. The diagnosis of a jugular paraganglioma may be delayed due to its atypical symptoms. We report a case of jugular paraganglioma presented as temporomandibular joint (TMJ) disorder

Clinical presentation: 29-years old woman with preliminary diagnosis probable atypical face pain. She experienced her pain for the first time 2 months prior to reference. Pain intensity was seven to eight out of ten points on Visual Analogue Scale, simple and combined analgetics and NSAIDs were ineffective. Pain was dull, aching, localized in the right temporal area with irradiation to the ipsilateral maxillar and periauricular areas. Pain was more intensive in the nighttime. Temporomandibular joint movement (maximum unassisted and assisted opening, right to left movements) also increased pain. Significant dilatation of right foramen jugularis with its right side destruction was detected by computed tomography with contrast. The radiological findings were consistent with those of a jugular paraganglioma.
\end{abstract}

Conclusion: This case confirms that in refractory headache and TMJ pain other reasons should be considered with rigorous investigation including neuroimaging.
Volume 6 Issue 3 - 2017

\author{
Julia Azimova,' Kirill Skorobogatykh,' \\ Konstantin Ishchenko,' Alexey Sergeev, ${ }^{1,2}$ \\ Eugene Klimov ${ }^{3,4}$ \\ 'University Headache Clinic, Russia \\ ${ }^{2}$ Department of Neuroscience, Sechenov First Moscow State \\ Medical University, Russia \\ Unniversity diagnostic laboratory, Russia \\ ${ }^{4}$ Faculty of Biology, Lomonosov Moscow State University, \\ Moscow, Russia
}

Correspondence: Julia Azimova, University Headache Clinic, Moscow, Russia, Email azimova.j@mail.com

Received: December 06, 2016 | Published: March 02, 2017

Keywords: jugular paraganlioma

Abbreviations: CT, computed tomography; TMJ, temporomandibular joint

\section{Introduction}

Jugular paraganlioma is a slow-growing tumor originating from the paraganglion cells. Its typical manifestations involve hearing loss, pulsatile tinnitus and lower cranial nerve deficits due to a mass effect. The diagnosis of a jugular paraganglioma may be delayed due to its atypical symptoms. We report a case of jugular paraganglioma presented as temporomandibular joint (TMJ) disorder.

\section{Case presentation}

29-years old woman was referred to University Headache Clinic with preliminary diagnosis probable atypical face pain. She experienced her pain for the first time 2 months prior to reference. Pain intensity was seven to eight out of ten points on Visual Analogue Scale, simple and combined analgetics and NSAIDs were ineffective. Her pain was dull, aching, localized in the right temporal area with irradiation to the ipsilateral maxillar and periauricular areas. Pain was more intensive in the nighttime. Temporomandibular joint movement (maximum unassisted and assisted opening, right to left movements) also increased pain. Before reference to our clinic the patient was treated with pregabalin (300 $\mathrm{mg}$ per day) and amitriptyline $(50 \mathrm{mg}$ per day) without significant improvement. On physical examination blood pressure was $120 / 70 \mathrm{mmHg}$, heart rate was 75 beats $/ \mathrm{min}$. Right temporal, maxillar, periauricular areas were visually without abnormalities. Temporomandibular joint mobility was limited; palpation of tight temporal and masseter muscles, the right pole was painful. Clicking noise during opening and closing movements was identified. Cutaneus allodynia was revealed in periauricular, temporal maxillare areas. Cranial nerves were intact.

Computed tomography (CT) with contrast (Ultravist 370, 100 $\mathrm{ml}$ ) was performed (Figure 1). Significant dilatation of right foramen jugularis with its right side destruction was detected: the left one size was $8 \times 7 \mathrm{~mm}$, the right one was $12 \times 14.6 \mathrm{~mm}$. In fossa jugularis we revealed a soft tissue mass with clear contours, homogeneous, destroying right wall of foramen jugularis. Enhanced-CT examination showed a significant enhancing mass. The radiological findings were consistent with those of a jugular paraganglioma. ${ }^{1}$

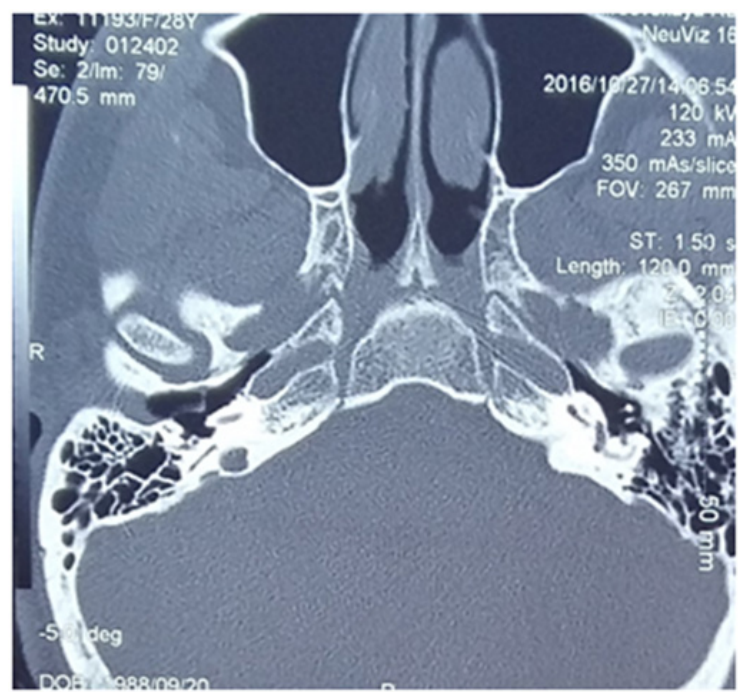

Figure I Results of computed tomography of the brain with contrast (Ultravist 370, $100 \mathrm{ml}$ ).

\section{Discussion}

Jugular paraganlioma is a rare benign tumor, which occurs in adults, typically between 40 and 60 years of age, more frequently among females. Typical symptoms are pulsatile tinnitus, hearing loss, hoarseness, dizziness. Headache occurs in $16 \%$ of the patients, otalgia - in $11 \% .^{2}$ Jugular paraganglioma presented as temporomandibular 
joint disorder is an extremely rare condition, we found only two similar cases in the literature. ${ }^{3,4}$ The patient meets the criteria of temporomandibular arthralgia (International RDC/TMD Consortium Network and Orofacial Pain Special Interest Group $)^{5}$ : 1. Pain of joint origin that is affected by jaw movement, function, or parafunction and replication of this pain occurs with provocation testing of TMJ. 2. Pain in the jaw, temple, in the ear or in front of ear. 3. Pain is modified with jaw movements, function, or parafunction. 4. Conformation of pain location in the area of TMJ. 5. Report of familiar pain with palpation of the lateral pole and maximum unassisted and assisted opening, right to left movements.

We assume some possible reasons for atypical paraganglioma presentation in our patient. Mass effect on facial nerve can lead to subclinical facial asymmetry, laterality of proprioception in the orofacial muscles and TMJ dysfunction. ${ }^{6}$ Paraganglioma rarely secrete various neuropeptide hormones, such as adrenocorticotropic hormone, and inflammatory cytokines, such as interleukin-6 (IL-6) inducing acute inflammatory reaction and pain. ${ }^{7}$

The patient was refractory to medication treatment: pain relievers, NSAIDs, pregabalin and amitriptyline were ineffective. This case confirms that in refractory headache and TMJ pain other reasons should be considered with rigorous investigation including neuroimaging.

\section{Acknowledgments}

The authors thank the subject for participation in this study.

\section{Conflicts of interest}

None.

\section{Funding}

None.

\section{References}

1. Rao AB, Koeller KK, Adair CF. From the archives of the AFIP. Paraganglioma of the head and neck: radiologic-pathologic correlation. Armed Forces Institute of Pathology. Radiographics. 1999;19(6):1605-1632.

2. Fayad JN, Keles B, Brackmann DE. Jugular foramen tumors: clinical characteristics and treatment outcomes. Otology \& Neurotology. 2010;31(2):299-305.

3. Terzic A, Becker M, Wissmeyer M, et al. 18F-DOPA PET/CT unravels malignant paraganglioma mimicking temporomandibular joint disorder. Dentomaxillofac Radiol. 2011;40(5):315-319.

4. Tashiro M, Nagase M, Nakajima T, et al. Malignant paraganglioma. Report of a case and review of the Japanese literature. J Craniomaxillofac Surg. 1988;16(7):324-329.

5. Schiffman E, Ohrbach R, Truelove E, et al. International RDC/TMD Consortium Network, International association for Dental Research; Orofacial Pain Special Interest Group, International Association for the Study of Pain. Diagnostic Criteria for Temporomandibular Disorders (DC/TMD) for Clinical and Research Applications: recommendations of the International RDC/TMD Consortium Network and Orofacial Pain Special Interest Group. J Oral Facial Pain Headache. 2014;28(1):6-27.

6. Frayne E, Coulson S, Adams R, et al. Laterality of proprioception in the Orofacial muscles and temporomandibular joint. Neurosci Lett. 2016;635:111-116

7. Sokabe A, Mizooka M, Sakemi R, et al. Systemic Inflammatory Syndrome Associated with a Case of Jugular Paraganglioma. Intern Med. 2016;55(15):2105-2018. 